\title{
Study on minerals status of dairy cows and their supplementation through area specific mineral mixture in the state of Jharkhand
}

\author{
B. M. Bhanderi ${ }^{1^{*}}$ (D, Ajay Goswami ${ }^{1}$, M. R. Garg ${ }^{1}$ and Saikat Samanta ${ }^{2}$
}

\begin{abstract}
Background: Deficiency of macro and micro-minerals in the ration of dairy cows adversely affects growth, milk production and reproduction efficiency. It is essential to examine mineral concentrations in feeds offered to dairy cows in practical farms.

Methods: Two villages from each taluka were selected at random for taking representative samples of feeds, forages and hair. Within the village, help was sought from village milk producers and district animal husbandry officer for identification of 4 to 5 farmers and collection of representative samples. All the samples were processed and analyzed for chemical composition as well as major macro and micro-minerals, using Inductively Coupled Plasma-Optical Emission Spectrometer.

Results: Ca content in wheat straw $(0.29 \%)$, crushed maize $(0.02 \%)$ and wheat bran $(0.12 \%)$ was found to be below the critical level $(0.30 \%)$. The $\mathrm{P}$ content in concentrate ingredients was high $(0.26-0.96 \%)$, but low in dry roughages $(0.06-0$. 12\%). Cereal straws (0.14\%) and grains (0.12\%) were deficient in Mg. Feeds and forages were found to be adequate in $\mathrm{K}$ (1.50\%). Cereals straws were found to be deficient in S (0.11\%). Greens were good source of Cu (12.02 ppm). Wheat straw was found to be low in Zn (18 ppm), but high in Mn (225 ppm) and Fe (509 ppm). Local grasses and azolla green were found to be rich source of Co (>1.00 ppm). Se $(0.63 \mathrm{ppm})$ was present in appreciable quantities in most of the feedstuffs.

Conclusions: From the present study, it was apparent that the feeds and forages available in the state of Jharkhand may not meet the requirements for $\mathrm{Ca}, \mathrm{P}, \mathrm{Mg}, \mathrm{Cu}, \mathrm{Zn}$ and $\mathrm{Co}$ in order to sustain a milk production of $\sim 10 \mathrm{~kg} /$ day. Therefore, it is necessary to supplement these deficient minerals through area specific mineral mixture in the ration of dairy cows for improving productivity and reproduction efficiency.
\end{abstract}

Keywords: Calcium, Phosphorus, Copper, Zinc, Feeds, Forage, Dairy cows

\section{Background}

Dietary nutrition plays a significant role in any livestock development programme and the optimum expression of genetic potential for milk production in dairy cows depend on adequate supply of nutrients. Micronutrients, particularly the mineral elements are considered to be inevitable for the normal metabolic

\footnotetext{
* Correspondence: bhanderi@nddb.coop

${ }^{1}$ Animal Nutrition Group, National Dairy Development Board (NDDB), Anand 388001, Gujarat, India

Full list of author information is available at the end of the article
}

and physiological processes of animal systems. The importance of minerals in regulating biological systems, growth, production and reproduction is well documented [1], however, livestock in India do not receive mineral/vitamin supplements except for common salt and calcite powder [2]. Hence, dairy cows depend on forages for their mineral requirements [3]. Garg et al. [4] and Bhanderi et al. [5] reported high incidences of forage and blood serum samples below the critical levels for $\mathrm{Cu}$ and $\mathrm{Zn}$. Miles and McDowell [6] demonstrated deficiency of $\mathrm{Cu}$ and $\mathrm{P}$ in the forage 
samples collected from the pasture. Soils from all over country have been being depleted for $\mathrm{Cu}, \mathrm{Zn}, \mathrm{P}$ and $\mathrm{S}$ in soil, plants and dairy cows [7]. The quantity of minerals, thus, present in forages may not be sufficient for optimum growth, milk yield and reproduction when those were fed to dairy cows [3]. In order to avoid macro and micro-minerals deficiency in the ration, a study on the assessment of mineral status of Holstein Friesian crossbred cows was undertaken in the state of Jharkhand.

\section{Methods}

\section{Sampling procedures}

Two villages from each taluka were selected at random for taking representative samples of feeds, forage and hair. Total area of Jharkhand state is 79,710 sq km distributed in to 24 districts, 211 blocks and 32,620 villages. The district is having annual rainfall of $1,400 \mathrm{~mm}$, latitude of $23^{0} 35^{\prime} \mathrm{N}$ and longitude of $85^{\circ} 33^{\prime} \mathrm{E}$. Atmospheric temperature ranges from 5 to $45^{\circ} \mathrm{C}$ during different seasons. In this study, two dimensional survey methods was adopted to map relevant mineral elements, by collecting feeds and forage samples from the representative villages, according to random sampling design based on conceptual landscape units [8]. Within the village, help was sought from village milk producers and district animal husbandry officer for identification of 4 to 5 farmers and collection of representative samples. The recorded parameters were number of livestock, land area, irrigation facilities, forage, other crops being grown, feeding practices followed, quantity of feeds, forage, dry roughage, concentrate feeds and mineral mixture offered to dairy cows. Representative samples of feeds, wet forages and dry roughages were collected from northern, eastern, western and southern directions of the selected villages. Further information regarding the amount and types of feeds and forages being offered to the dairy cows, actual rate of daily feed intake, number of milking cows and milk yield were collected from individual farmer. Daily feed intakes were monitored through the INAPH software. INAPH or Information Network for Animal Productivity and Health, a windows based Internet linked application, developed by National Dairy Development Board of India to assess the prevailing status of the nutrient provision to the animal against the animal's nutrient requirements [9]. Both these sets of information are used to work out a least cost ration with the available feed resources and an area specific mineral mixture [10]. Total intake was compared against the requirements on dry matter basis [11], so as to identify quantitative deficiency, adequacy or even excess. With the help of INAPH software, status of metabolizable energy, protein intake and mineral status of dairy cows reared under field conditions was assessed [9].

\section{Sample preparation and analytical methods}

Weekly basis composite samples of wet forages, cereal straws/dry forages, concentrate feed ingredients and the compound cattle feed (concentrate mixture) were collected from all over the surveyed area. Surveyed area used for the places/villages in the district from where representative samples of feed, wet forage, dry roughage and hair samples were collected. Wet forage samples were dried in hot air oven at $100{ }^{\circ} \mathrm{C}$ for $24 \mathrm{~h}$ and subsequently ground $(1 \mathrm{~mm})$. Ground samples of concentrate and forages were stored in airtight bags until analysis. The samples of feeds, wet forages and dry roughages were analyzed for crude protein $(\mathrm{CP})$, ether extract (EE), crude fibre (CF) and acid insoluble ash (AIA) as per AOAC [12] and for neutral detergent fibre and acid detergent fibre as per Van Soest et al. [13]. All the samples were also analyzed for calcium $(\mathrm{Ca})$, phosphorus $(\mathrm{P})$, magnesium $(\mathrm{Mg})$, sulphur $(\mathrm{S})$, sodium $(\mathrm{Na})$, potassium $(\mathrm{K})$, copper $(\mathrm{Cu})$, zinc $(\mathrm{Zn})$, manganese $(\mathrm{Mn})$, iron $(\mathrm{Fe})$, cobalt $(\mathrm{Co})$, selenium (Se) and molybdenum (Mo), using Inductively Coupled Plasma-Optical Emission Spectrometer (Perkin-Elmer, OPTIMA-3300 RL).

The word "critical" is used in this article to note a concentration in feedstuffs below (or above with excesses) what is considered the requirement for dairy cow [14]. This assumes the expected consumption as estimated by the NRC [11]. Total grams/milligrams of minerals consumed per day determine the true adequacy of a mineral, not the forage concentration [15].

\section{Statistical analysis}

The data were analyzed statistically as per the Snedecor and Cochran [16], with the help of SPSS package programme (SPSS 9.00 software for Windows, SPSS Inc., Chicago, IL).

\section{Results and discussion}

Crop residues were found to be the main source of dry roughages in the ration of dairy cows. It was noticed that some of the milk producers fed cultivated forages like, maize (Zea mays), jowar green (Sorghum bicolor) etc. Some milk producers offered crushed maize, crushed wheat alone or their mixture. Feeding mustard oil cake, wheat bran, linseed cake and maize germ cake was also observed in some parts of state. Those milk producers, who didn't feed concentrate ingredients, were feeding compound cattle feed depending on the level of milk production. The use of common salt and mineral mixture supplementation was not a common practice in the surveyed area, except for therapeutic purpose on prescription by veterinary officer. 


\section{Chemical composition of feeds and forages}

The feed and forage samples collected from surveyed area were analyzed for chemical composition (Table 1). Mustard oil cake was a good source of protein (37.98\% crude protein), whereas, linseed meal contained 32.84\% crude protein (CP). Amongst wet forage azolla (22.18\%) had the highest CP content followed by maize (7.24\%) and para grass (5.35\%). Cereal straws/ dry forages were low in CP content, except gotars of Bengal gram (Cicer arietinum), arhar/pigeon pea (Cajanus cajan) and urd (Phaseolus mungo). Neutral detergent fibre (NDF) and acid detergent fibre (ADF) content was found to be highest in dry roughages. The data on proximate composition of the feedstuffs are in agreement [17-19].

\section{Nutritional status of lactating dairy cows}

In order of priority, available good quality feed resources are first allocated to lactating dairy cows followed by dry pregnant, dry, heifers, growing calves and non-productive cows. In the surveyed area our observation indicates that metabolizable energy were in excess in the ration of more than $65 \%$ of cows, crude protein was deficient in the diet of more than $85 \%$ of dairy cows, $\mathrm{Ca}$ and $\mathrm{P}$ were deficient in the ration of about $70 \%$ of the cows because of inadequate mineral mixture supplementation. Milk producers in most of the developing countries often do not feed adequate quantities of mineral mixture to their dairy cows due to non-availability, lack of knowledge on the benefits of feeding mineral mixtures [20]. In view of this, there is an urgent need to popularize supplementation of mineral mixture in the ration of dairy cows for improving production and reproduction efficiency [21]. In developing countries, Garg and Sherasia [22] reported that daily milk production of dairy cows is low, as compared to developed nations. Similarly, Garg et al. [23] reported that age at first calving and inter-calving intervals is higher in Zebu dairy cows, affecting life time productivity in developing countries.

\section{Macro-minerals profile of feeds and forages}

The straws of wheat and paddy were the main roughage sources in the surveyed area (Tables 2 and 3). The average $\mathrm{Ca}$ content ranged from 0.29 to $1.17 \%$ in roughages as compared to 0.02 to $0.68 \%$ in concentrate feed ingredients. These findings are similar to the findings of Ramana et al. [24]. The similar findings were also reported by Udar et al. [25] and Bhanderi et al. [26]. P content in concentrates (0.17 to $0.96 \%)$ was higher than dry roughages (0.06 to $0.13 \%)$. Crushed grains were low in $\mathrm{Mg}$ as compared to cakes (Table 3). S content was

Table 1 Chemical composition of feed and forages in Jharkhand state (on DM basis)

\begin{tabular}{|c|c|c|c|c|c|c|}
\hline Feed item & $\mathrm{CP}(\%)$ & $\mathrm{EE}(\%)$ & CF (\%) & NDF (\%) & ADF (\%) & AIA (\%) \\
\hline \multicolumn{7}{|l|}{ Grains/seeds } \\
\hline Crushed wheat (4), Triticum aestivum & $11.95 \pm 0.43$ & $1.98 \pm 0.09$ & $1.25 \pm 0.07$ & $14.82 \pm 0.67$ & $5.63 \pm 0.11$ & $0.23 \pm 0.04$ \\
\hline Crushed maize (5), Zea mays & $8.65 \pm 0.12$ & $3.48 \pm 0.09$ & $1.92 \pm 0.08$ & $15.62 \pm 0.12$ & $4.59 \pm 0.04$ & $0.26 \pm 0.05$ \\
\hline \multicolumn{7}{|l|}{ Brans/cakes/chunnies } \\
\hline Wheat bran (5), Triticum aestivum & $15.08 \pm 0.29$ & $2.08 \pm 0.08$ & $16.78 \pm 0.62$ & $62.38 \pm 0.98$ & $18.93 \pm 0.68$ & $3.48 \pm 0.11$ \\
\hline Mustard oil cake (6), Brassica campestris & $37.98 \pm 1.25$ & $6.77 \pm 0.34$ & $7.18 \pm 0.23$ & $26.66 \pm 0.69$ & $17.84 \pm 0.39$ & $2.46 \pm 0.18$ \\
\hline Linseed meal (4), Linum usitatissimum & $32.84 \pm 0.86$ & $0.62 \pm 0.08$ & $9.5 \pm 0.34$ & $24.18 \pm 0.27$ & $18.67 \pm 0.19$ & $2.05 \pm 0.18$ \\
\hline Sesame cake (3), Sesamum indicum & $29.80 \pm 1.13$ & $8.46 \pm 0.68$ & $8.90 \pm 0.91$ & $27.44 \pm 0.86$ & $16.88 \pm 0.48$ & $3.11 \pm 0.16$ \\
\hline Gram chunni (3), Cicer arietinum & $18.80 \pm 1.24$ & $3.56 \pm 0.32$ & $11.38 \pm 0.23$ & $32.18 \pm 2.48$ & $18.68 \pm 0.93$ & $0.78 \pm 0.11$ \\
\hline $\begin{array}{l}\text { Masoor chunni + arhar chunni (4), Lens } \\
\text { culinaris + Cajanus cajan }\end{array}$ & $20.85 \pm 0.67$ & $1.90 \pm 0.34$ & $12.88 \pm 0.22$ & $28.38 \pm 2.78$ & $17.82 \pm 0.82$ & $1.86 \pm 0.18$ \\
\hline \multicolumn{7}{|l|}{ Green fodder/grasses } \\
\hline Maize green (5), Zea mays & $7.24 \pm 0.11$ & $1.25 \pm 0.08$ & $27.44 \pm 0.18$ & $52.68 \pm 3.22$ & $33.17 \pm 1.23$ & $2.36 \pm 0.11$ \\
\hline Para grass (4), Brachiaria mutica & $5.35 \pm 0.16$ & $1.65 \pm 0.11$ & $30.44 \pm 0.46$ & $64.89 \pm 3.88$ & $31.33 \pm 1.44$ & $2.90 \pm 0.19$ \\
\hline Local green grasses (6) & $4.65 \pm 0.09$ & $1.93 \pm 0.12$ & $34.68 \pm 0.23$ & $58.66 \pm 2.88$ & $29.89 \pm 2.08$ & $3.11 \pm 0.18$ \\
\hline Azolla green (6), Azolla pinnata & $22.18 \pm 1.45$ & $3.89 \pm 0.48$ & $12.7 \pm 0.17$ & $36.78 \pm 1.88$ & $23.18 \pm 1.18$ & $2.56 \pm 0.13$ \\
\hline \multicolumn{7}{|l|}{ Straws/gotars } \\
\hline Wheat straw (6), Triticum aestivum & $2.15 \pm 0.11$ & $0.69 \pm 0.06$ & $38.44 \pm 2.34$ & $78.58 \pm 2.33$ & $56.12 \pm 3.12$ & $5.44 \pm 0.21$ \\
\hline Paddy straw (5), Oryza sativa & $3.44 \pm 0.18$ & $1.11 \pm 0.15$ & $42.32 \pm 1.89$ & $68.75 \pm 2.44$ & $44.78 \pm 3.19$ & $5.18 \pm 0.23$ \\
\hline Gram gotar (4); Cicer arietinum & $8.11 \pm 0.11$ & $2.01 \pm 0.19$ & $34.67 \pm 1.88$ & $54.89 \pm 3.19$ & $38.67 \pm 2.18$ & $3.90 \pm 0.21$ \\
\hline Arhar gotar (6), Cajanus cajan & $8.75 \pm 0.19$ & $1.89 \pm 0.18$ & $33.88 \pm 2.15$ & $56.14 \pm 2.28$ & $40.18 \pm 3.88$ & $4.18 \pm 0.28$ \\
\hline Urd gotar (4), Phaseolus mungo & $9.44 \pm 0.34$ & $1.44 \pm 0.12$ & $29.44 \pm 1.09$ & $58.33 \pm 1.90$ & $42.23 \pm 2.19$ & $4.88 \pm 0.31$ \\
\hline
\end{tabular}

Figures in the parentheses indicate no. of samples analysed 
Table 2 Macro-minerals content in dry and wet forages (on DM basis)

\begin{tabular}{|c|c|c|c|c|c|c|}
\hline Particular & $\mathrm{Ca}$ & $P$ & $\mathrm{Mg}$ & S & K & $\mathrm{Na}$ \\
\hline Critical level ${ }^{*}$ & $<0.30 \%$ & $<0.25 \%$ & $<0.20$ & $<0.20 \%$ & $<0.9 \%$ & $<0.06 \%$ \\
\hline Wheat straw (69), Triticum aestivum & $0.29 \pm 0.02$ & $0.06 \pm 0.00$ & $0.12 \pm 0.01$ & $0.12 \pm 0.01$ & $1.43 \pm 0.08$ & $0.04 \pm 0.01$ \\
\hline Paddy straw (123), Oryza sativa & $0.36 \pm 0.01$ & $0.08 \pm 0.00$ & $0.17 \pm 0.00$ & $0.11 \pm 0.00$ & $1.71 \pm 0.04$ & $0.12 \pm 0.01$ \\
\hline Gram straw (4), Cicer arietinum & $1.04 \pm 0.02$ & $0.11 \pm 0.01$ & $0.26 \pm 0.01$ & $0.21 \pm 0.01$ & $2.05 \pm 0.09$ & $0.07 \pm 0.01$ \\
\hline Masoor straw (3), Lens culinaris & $1.17 \pm 0.03$ & $0.11 \pm 0.01$ & $0.23 \pm 0.02$ & $0.15 \pm 0.02$ & $2.01 \pm 0.04$ & $0.04 \pm 0.01$ \\
\hline Arhar straw (6), Cajanus cajan & $1.12 \pm 0.03$ & $0.13 \pm 0.00$ & $0.27 \pm 0.01$ & $0.17 \pm 0.00$ & $1.45 \pm 0.1$ & $0.03 \pm 0.01$ \\
\hline Urd straw (4), Phaseolus mungo & $0.94 \pm 0.02$ & $0.12 \pm 0.31$ & $1.69 \pm 0.46$ & $2.08 \pm 0.68$ & $3.70 \pm 0.81$ & $2.51 \pm 0.88$ \\
\hline Maize green (22), Zea mays & $0.58 \pm 0.05$ & $0.33 \pm 0.03$ & $0.35 \pm 0.02$ & $0.26 \pm 0.02$ & $3.33 \pm 0.31$ & $0.03 \pm 0.01$ \\
\hline Sudan grass (11), Sorghum sudanense & $0.58 \pm 0.05$ & $0.25 \pm 0.01$ & $0.32 \pm 0.02$ & $0.19 \pm 0.00$ & $2.28 \pm 0.06$ & $0.06 \pm 0.00$ \\
\hline Cowpea green (4), Vigna sinensis & $2.01 \pm 0.2$ & $0.33 \pm 0.03$ & $0.46 \pm 0.04$ & $0.34 \pm 0.01$ & $2.27 \pm 0.16$ & $0.03 \pm 0.01$ \\
\hline Azolla green (12), Azolla pinnata & $1.50 \pm 0.08$ & $0.84 \pm 0.23$ & $0.41 \pm 0.07$ & $0.40 \pm 0.04$ & $2.84 \pm 0.46$ & $0.70 \pm 0.13$ \\
\hline Bajri green (4), Pennisetum typhoides & $0.59 \pm 0.06$ & $0.45 \pm 0.04$ & $0.30 \pm 0.01$ & $0.21 \pm 0.02$ & $2.59 \pm 0.42$ & $0.01 \pm 0.01$ \\
\hline Para grass (4), Brachiaria mutica & $0.63 \pm 0.07$ & $0.34 \pm 0.02$ & $0.19 \pm 0.02$ & $0.22 \pm 0.01$ & $5.05 \pm 0.76$ & $0.01 \pm 0.02$ \\
\hline Local green grasses (48) & $0.88 \pm 0.07$ & $0.33 \pm 0.02$ & $0.41 \pm 0.03$ & $0.38 \pm 0.02$ & $3.16 \pm 0.15$ & $0.12 \pm 0.02$ \\
\hline
\end{tabular}

Figures in the parentheses indicate no. of samples analysed

*Concentrations below which are low or considered to be deficient (McDowell et al., 1993), based on requirements for cattle [11]

Table 3 Macro-minerals content in concentrate feed ingredients (on DM basis)

\begin{tabular}{|c|c|c|c|c|c|c|}
\hline Particular & $\mathrm{Ca}$ & $P$ & $\mathrm{Mg}$ & S & K & $\mathrm{Na}$ \\
\hline Critical level ${ }^{*}$ & $<0.30 \%$ & $<0.25 \%$ & $<0.20 \%$ & $<0.20 \%$ & $<0.9 \%$ & $<0.06 \%$ \\
\hline Crushed maize (25), Zea mays & $0.02 \pm 0.00$ & $0.26 \pm 0.01$ & $0.10 \pm 0.00$ & $0.11 \pm 0.00$ & $0.37 \pm 0.01$ & $0.01 \pm 0.00$ \\
\hline Wheat bran (81), Triticum aestivum & $0.12 \pm 0.02$ & $0.84 \pm 0.03$ & $0.33 \pm 0.01$ & $0.20 \pm 0.00$ & $1.06 \pm 0.03$ & $0.02 \pm 0.00$ \\
\hline Mustard oil cake (54), Brassica campestris & $0.68 \pm 0.03$ & $0.96 \pm 0.03$ & $0.40 \pm 0.02$ & $1.26 \pm 0.05$ & $1.12 \pm 0.04$ & $0.02 \pm 0.00$ \\
\hline Cattle feed (21) & $0.99 \pm 0.1$ & $1.41 \pm 0.06$ & $0.78 \pm 0.03$ & $0.44 \pm 0.03$ & $1.26 \pm 0.05$ & $0.65 \pm 0.06$ \\
\hline Gram chunni (9), Cicer arieninum & $0.63 \pm 0.14$ & $0.25 \pm 0.02$ & $0.33 \pm 0.08$ & $0.13 \pm 0.01$ & $0.73 \pm 0.03$ & $0.02 \pm 0.00$ \\
\hline Rice bran (15), Oryza sativa & $0.11 \pm 0.01$ & $0.46 \pm 0.05$ & $0.29 \pm 0.02$ & $0.11 \pm 0.01$ & $0.61 \pm 0.07$ & $0.01 \pm 0.00$ \\
\hline $\begin{array}{l}\text { Maize + wheat mixture (17), Zea mays + } \\
\text { Triticum aestivum }\end{array}$ & $0.20 \pm 0.07$ & $0.36 \pm 0.02$ & $0.20 \pm 0.03$ & $0.17 \pm 0.02$ & $0.61 \pm 0.06$ & $0.02 \pm 0.01$ \\
\hline Crushed wheat (12), Triticum aestivum & $0.06 \pm 0.01$ & $0.31 \pm 0.01$ & $0.12 \pm 0.00$ & $0.15 \pm 0.01$ & $0.41 \pm 0.01$ & $0.00 \pm 0.00$ \\
\hline Wheat grain (5), Triticum aestivum & $0.04 \pm 0.00$ & $0.30 \pm 0.01$ & $0.11 \pm 0.00$ & $0.17 \pm 0.01$ & $0.38 \pm 0.01$ & $0.01 \pm 0.00$ \\
\hline Gram flour (15), Cicer arietinum & $0.09 \pm 0.02$ & $0.29 \pm 0.02$ & $0.12 \pm 0.01$ & $0.17 \pm 0.02$ & $0.74 \pm 0.08$ & $0.01 \pm 0.00$ \\
\hline Kesari dal (3), Lathyrus sativus & $0.13 \pm 0.00$ & $0.28 \pm 0.00$ & $0.14 \pm 0.00$ & $0.18 \pm 0.01$ & $0.73 \pm 0.09$ & $0.02 \pm 0.00$ \\
\hline Linseed cake (6), Linum usitatissimum & $0.49 \pm 0.04$ & $0.58 \pm 0.02$ & $0.38 \pm 0.01$ & $0.35 \pm 0.04$ & $0.92 \pm 0.04$ & $0.02 \pm 0.00$ \\
\hline Maize grain (5), Zea mays & $0.01 \pm 0.00$ & $0.27 \pm 0.01$ & $0.10 \pm 0.01$ & $0.11 \pm 0.01$ & $0.39 \pm 0.01$ & $0.01 \pm 0.00$ \\
\hline Masoor chunni (4), Lens culinaris & $0.37 \pm 0.02$ & $0.21 \pm 0.06$ & $0.28 \pm 0.02$ & $0.10 \pm 0.01$ & $0.50 \pm 0.02$ & $0.04 \pm 0.00$ \\
\hline $\begin{array}{l}\text { Masoor + arhar mix chunni (6), Lens culinaris + } \\
\text { Cajanus cajan }\end{array}$ & $0.43 \pm 0.01$ & $0.17 \pm 0.03$ & $0.21 \pm 0.01$ & $0.12 \pm 0.01$ & $0.83 \pm 0.15$ & $0.04 \pm 0.01$ \\
\hline Sesame cake (6), Sesamum indicum & $0.64 \pm 0.18$ & $0.77 \pm 0.04$ & $0.45 \pm 0.02$ & $0.54 \pm 0.06$ & $1.12 \pm 0.04$ & $0.05 \pm 0.01$ \\
\hline Jowar green (2), Sorghum bicolor & $0.43 \pm 0.05$ & $0.29 \pm 0.02$ & $0.27 \pm 0.02$ & $0.20 \pm 0.01$ & $2.01 \pm 0.16$ & $0.01 \pm 0.00$ \\
\hline Wheat flour (7), Triticum aestivum & $0.06 \pm 0.01$ & $0.30 \pm 0.01$ & $0.13 \pm 0.00$ & $0.18 \pm 0.00$ & $0.41 \pm 0.02$ & $0.03 \pm 0.00$ \\
\hline Bengal gram (2), Cicer arietinum & $0.23 \pm 0.02$ & $0.49 \pm 0.04$ & $0.18 \pm 0.01$ & $0.27 \pm 0.02$ & $1.07 \pm 0.03$ & $0.03 \pm 0.01$ \\
\hline Maize cake (2), Zea mays & $0.07 \pm 0.01$ & $0.28 \pm 0.02$ & $0.06 \pm 0.01$ & $0.24 \pm 0.02$ & $0.13 \pm 0.01$ & $0.02 \pm 0.01$ \\
\hline
\end{tabular}

Figures in the parentheses indicate no. of samples analysed

*Concentrations below which are low or considered to be deficient (McDowell et al., 1993), based on requirements for cattle [11] 
Table 4 Micro-minerals content in dry and wet forages (on DM basis)

\begin{tabular}{|c|c|c|c|c|c|c|c|}
\hline Particular & $\mathrm{Cu}$ & $\mathrm{Zn}$ & $\mathrm{Mn}$ & $\mathrm{Fe}$ & Co & Mo & Se \\
\hline Critical level ${ }^{*}$ & $<8$ ppm & $<30$ ppm & $<40$ ppm & $<50$ ppm & $<0.10$ ppm & $>6$ ppm & $<0.2 \mathrm{ppm}$ \\
\hline Wheat straw (69), Triticum aestivum & $2.46 \pm 0.21$ & $18.00 \pm 2.85$ & $225 \pm 58.7$ & $509 \pm 62$ & $0.32 \pm 0.06$ & $0.56 \pm 0.03$ & $1.33 \pm 0.21$ \\
\hline Paddy straw (123), Oryza sativa & $2.99 \pm 0.14$ & $39.79 \pm 1.17$ & $663 \pm 28.2$ & $851 \pm 82$ & $0.73 \pm 0.06$ & $0.21 \pm 0.02$ & $0.78 \pm 0.00$ \\
\hline Gram straw (4), Cicer arietinum & $5.93 \pm 0.02$ & $17.20 \pm 1.28$ & $75 \pm 12.3$ & $1,015 \pm 136$ & $0.56 \pm 0.06$ & $0.87 \pm 0.11$ & $0.64 \pm 0.07$ \\
\hline Masoor straw (3), Lens culinaris & $7.01 \pm 0.33$ & $20.49 \pm 3.33$ & $52 \pm 3.1$ & $988 \pm 93$ & $0.56 \pm 0.04$ & $0.28 \pm 0.08$ & $0.36 \pm 0.05$ \\
\hline Arhar straw (6), Cajanus cajan & $7.34 \pm 0.43$ & $24.08 \pm 1.06$ & $75 \pm 2.6$ & $1,124 \pm 65$ & $0.70 \pm 0.05$ & $0.72 \pm 0.05$ & $0.54 \pm 0.05$ \\
\hline Urd straw (4), Phaseolus mungo & $10.42 \pm 0.09$ & $24.97 \pm 5.10$ & $31 \pm 6.0$ & $209 \pm 33$ & $0.11 \pm 0.02$ & $0.36 \pm 0.06$ & $0.30 \pm 0.08$ \\
\hline Maize green (22), Zea mays & $10.22 \pm 1.26$ & $37.91 \pm 2.07$ & $179 \pm 44.1$ & $1,217 \pm 189$ & $0.72 \pm 0.11$ & $1.96 \pm 0.30$ & $0.71 \pm 0.10$ \\
\hline Sudan grass (11), Sorghum sudanense & $09.38 \pm 1.00$ & $31.36 \pm 1.58$ & $241 \pm 64.7$ & $1,018 \pm 105$ & $0.51 \pm 0.07$ & $0.29 \pm 0.10$ & $0.15 \pm 0.05$ \\
\hline Cowpea green (4), Vigna sinensis & $10.42 \pm 1.08$ & $39.43 \pm 2.24$ & $135 \pm 12.6$ & $1,088 \pm 105$ & $0.55 \pm 0.05$ & $2.52 \pm 0.39$ & $1.05 \pm 0.12$ \\
\hline Azolla green (12), Azolla pinnata & $11.38 \pm 2.16$ & $131.23 \pm 40.55$ & $4,068 \pm 826$ & $2,599 \pm 43$ & $17.67 \pm 2.92$ & $5.06 \pm 1.25$ & $0.52 \pm 0.10$ \\
\hline Bajri green (4), Pennisetum typhoides & $19.19 \pm 1.34$ & $28.46 \pm 2.78$ & $45 \pm 7.1$ & $465 \pm 197$ & $0.25 \pm 0.08$ & $1.35 \pm 0.10$ & $0.76 \pm 0.10$ \\
\hline Para grass (4), Brachiaria mutica & $13.14 \pm 0.94$ & $29.29 \pm 3.20$ & $55 \pm 1.5$ & $426 \pm 39$ & $0.26 \pm 0.03$ & $0.08 \pm 0.47$ & $0.08 \pm 0.02$ \\
\hline Local green grasses (48) & $10.43 \pm 0.93$ & $42.83 \pm 3.22$ & $254 \pm 35.5$ & $1,972 \pm 506$ & $1.33 \pm 0.38$ & $0.08 \pm 0.02$ & $0.48 \pm 0.05$ \\
\hline Jowar green (4), Sorghum bicolor & $09.69 \pm 1.85$ & $22.77 \pm 4.39$ & $115 \pm 34.4$ & $548 \pm 99$ & $0.35 \pm 0.06$ & $0.07 \pm 0.00$ & $0.28 \pm 0.05$ \\
\hline
\end{tabular}

Figures in the parentheses indicate no. of samples analyzed

${ }^{*}$ Concentrations below which are low or considered to be deficient (McDowell et al., 1993), based on requirements for cattle [11]

Table 5 Micro-minerals content in concentrate feed ingredients (on DM basis)

\begin{tabular}{|c|c|c|c|c|c|c|c|}
\hline Particular & $\mathrm{Cu}$ & $\mathrm{Zn}$ & $\mathrm{Mn}$ & $\mathrm{Fe}$ & Co & Mo & Se \\
\hline Critical level* & $<8 \mathrm{ppm}$ & $<30$ ppm & $<40$ ppm & $<50$ ppm & $<0.10$ ppm & $>6$ ppm & $<0.2 \mathrm{ppm}$ \\
\hline Crushed maize (25), Zea mays & $02.81 \pm 0.26$ & $21.31 \pm 0.49$ & $11 \pm 0.5$ & $185 \pm 38$ & $0.10 \pm 0.01$ & $1.63 \pm 0.07$ & $1.53 \pm 0.12$ \\
\hline Wheat bran (81), Triticum aestivum & $11.77 \pm 0.46$ & $75.21 \pm 2.11$ & $100 \pm 6.7$ & $475 \pm 62$ & $0.23 \pm 0.03$ & $0.46 \pm 0.05$ & $1.35 \pm 0.16$ \\
\hline Mustard oil cake (54), Brassica campestris & $09.97 \pm 0.44$ & $62.32 \pm 1.60$ & $70 \pm 5.7$ & $1,019 \pm 95$ & $0.48 \pm 0.05$ & $2.57 \pm 0.06$ & $0.60 \pm 0.04$ \\
\hline Cattle feed (21) & $12.71 \pm 0.75$ & $124.29 \pm 11.08$ & $192 \pm 12.5$ & $1,147 \pm 120$ & $1.21 \pm 0.14$ & $0.95 \pm 0.15$ & $0.28 \pm 0.04$ \\
\hline Gram chunni (9), Cicer arietinum & $12.25 \pm 2.03$ & $29.33 \pm 2.12$ & $51 \pm 6.2$ & $608 \pm 96$ & $0.38 \pm 0.04$ & $3.06 \pm 0.15$ & $0.02 \pm 0.29$ \\
\hline Rice bran (15), Oryza sativa & $16.48 \pm 2.04$ & $48.06 \pm 5.15$ & $200 \pm 14.6$ & $791 \pm 76$ & $0.32 \pm 0.06$ & $0.45 \pm 0.05$ & $0.91 \pm 0.14$ \\
\hline $\begin{array}{l}\text { Maize + wheat mixture (17), Zea mays + } \\
\text { Triticum aestivum }\end{array}$ & $8.57 \pm 0.59$ & $35.77 \pm 2.61$ & $43 \pm 5.7$ & $713 \pm 103$ & $0.41 \pm 0.07$ & $0.34 \pm 0.33$ & $1.58 \pm 0.25$ \\
\hline Crushed wheat (12), Triticum aestivum & $4.17 \pm 0.28$ & $29.31 \pm 1.66$ & $30 \pm 1.7$ & $217 \pm 51$ & $0.18 \pm 0.03$ & $0.68 \pm 0.03$ & $0.77 \pm 0.17$ \\
\hline Wheat grain (5), Triticum aestivum & $4.97 \pm 0.35$ & $31.16 \pm 1.95$ & $47.5 \pm 3.2$ & $106 \pm 11$ & $0.18 \pm 0.03$ & $0.03 \pm 0.00$ & $0.68 \pm 0.18$ \\
\hline Gram flour (15), Cicer arietinum & $06.89 \pm 1.18$ & $30.69 \pm 3.07$ & $24 \pm 3.2$ & $221 \pm 70$ & $0.30 \pm 0.05$ & $0.77 \pm 2.45$ & $1.03 \pm 0.08$ \\
\hline Kesari dal (3), Lathyrus sativus & $6.54 \pm 0.38$ & $32.36 \pm 1.15$ & $43 \pm 4.0$ & $999 \pm 82$ & $0.59 \pm 0.01$ & $0.70 \pm 0.23$ & $0.40 \pm 0.11$ \\
\hline Linseed cake (6), Linum usitatissimum & $20.05 \pm 1.51$ & $57.82 \pm 1.56$ & $70 \pm 4.9$ & $1,715 \pm 97$ & $1.32 \pm 0.08$ & $0.97 \pm 0.28$ & $0.64 \pm 0.15$ \\
\hline Maize grain (5), Zea mays & $3.60 \pm 0.68$ & $20.23 \pm 2.65$ & $10 \pm 8.6$ & $278 \pm 34$ & $0.18 \pm 0.05$ & $1.84 \pm 0.23$ & $0.21 \pm 0.11$ \\
\hline Masoor chunni (4), Lens culinaris & $10.70 \pm 0.27$ & $44.67 \pm 1.61$ & $76 \pm 3.1$ & $1,992 \pm 10$ & $1.29 \pm 0.03$ & $0.03 \pm 0.00$ & $0.68 \pm 0.18$ \\
\hline $\begin{array}{l}\text { Masoor + arhar mix chunni (6), Lens } \\
\text { culinaris + Cajanus cajan }\end{array}$ & $13.60 \pm 0.31$ & $40.97 \pm 2.81$ & $27 \pm 1.5$ & $388 \pm 73$ & $0.30 \pm 0.01$ & $0.77 \pm 2.45$ & $0.86 \pm 0.12$ \\
\hline Sesame cake (6), Sesamum indicum & $21.88 \pm 1.04$ & $78.42 \pm 1.72$ & $60 \pm 2.6$ & $1,098 \pm 90$ & $0.61 \pm 0.05$ & $0.70 \pm 0.23$ & $0.40 \pm 0.11$ \\
\hline Wheat flour (7), Triticum aestivum & $5.25 \pm 0.04$ & $31.81 \pm 0.42$ & $61 \pm 12.4$ & $199 \pm 17$ & $0.13 \pm 0.02$ & $0.97 \pm 0.28$ & $0.75 \pm 0.26$ \\
\hline Bengal gram (2), Cicer arietinum & $9.91 \pm 0.68$ & $48.88 \pm 2.65$ & $30 \pm 8.6$ & $173 \pm 34$ & $0.36 \pm 0.05$ & $0.69 \pm 0.23$ & $0.21 \pm 0.11$ \\
\hline Maize germ cake (2), Zea mays & $4.04 \pm 0.38$ & $24.61 \pm 3.65$ & $5 \pm 0.6$ & $158 \pm 24$ & $0.02 \pm 0.00$ & $0.19 \pm 0.03$ & $0.01 \pm 0.00$ \\
\hline
\end{tabular}


Table 6 Mineral content in hair samples of dairy cows

\begin{tabular}{lllllllllll}
\hline Particular & $\mathrm{Ca}$ & $\mathrm{P}$ & $\mathrm{Mg}$ & $\mathrm{S}$ & $\mathrm{Na}$ & $\mathrm{K}$ & $\mathrm{Cu}$ & $\mathrm{Zn}$ & $\mathrm{Mn}$ \\
$(\%)$ & $(\%)$ & $(\%)$ & $(\%)$ & $(\%)$ & $(\%)$ & $(\mathrm{ppm})$ & $\begin{array}{l}\text { Se (ppm) } \\
(\mathrm{ppm})\end{array}$ \\
\hline Hair samples & 0.39 & 0.09 & 0.31 & 2.99 & 0.14 & 0.83 & 06.77 & 63.51 & 207.96 & 3.48 \\
$(n=20)$ & \pm 0.04 & \pm 0.01 & \pm 0.06 & \pm 0.09 & \pm 0.03 & \pm 0.10 & \pm 0.45 & \pm 4.05 & \pm 50.35 & \pm 0.33 \\
\hline
\end{tabular}

found below critical level $(<0.20 \%)$ in most of the straws and crushed grain [27]. Higher $\mathrm{K}$ level in wet forages may be due to its selective uptake from the soil and regular application of potash fertilizer in the soil [2]. Na content was low in some of the feedstuffs (Tables 2 and 3 ).

\section{Micro-minerals profile of feeds and forages}

$\mathrm{Cu}$ content was found below the critical level $(<8 \mathrm{ppm})$ in wheat straw, paddy straw and crushed grains (Tables 4 and 5). Zn content was below critical level $(<30 \mathrm{ppm})$ in all the straws except paddy straw [27\}. Wet forages and cakes were found to be a better source of $\mathrm{Zn}$ as compared to crushed grains (Tables 4 and 5). The Mn levels in the state ranged from $36.88-662.56 \mathrm{ppm}$ in straws, 45.21-254.29 ppm in wet forages and 4.97200.17 ppm in concentrate ingredients. Average Fe content was $1037 \mathrm{ppm}$ in roughage and $651 \mathrm{ppm}$ in concentrates, showing adequacy of this mineral. Youssef et al. [28] and Yadav et al. [29] reported high Fe levels in forages. Co content in most of the feeds and forages ranged from $0.02 \mathrm{ppm}$ to $1.33 \mathrm{ppm}$, except in azolla green in which it was as high as 17.67 (Tables 4 and 5). Se and Mo content were adequate in all the feeds and forages. High levels of $\mathrm{Mo}(>2 \mathrm{ppm})$ in forages could interfere with $\mathrm{Cu}$ metabolism. The Mo levels as estimated in the samples of crop residues were within the safe limit. Most of the feedstuffs contained Mo level within the safe limit and gave $\mathrm{Cu}$ :Mo ratio wider than 5.0. Mo has gained more importance recently in animal nutrition, because of its inhibitory role on the other trace elements, particularly $\mathrm{Cu}$. Suttle [30] stated that a $\mathrm{Cu}$ :Mo ratio below 2.0 would be expected to cause conditioned $\mathrm{Cu}$ deficiency in dairy cows. Mo level at 5 to $6 \mathrm{ppm}$ inhibits $\mathrm{Cu}$ storage and produce signs of molybdenosis [31]. Even $2 \mathrm{ppm}$ or less can be toxic, if forage $\mathrm{Cu}$ is sufficiently low [32]. In case of ruminants, Mo reacts with sulphur in the rumen and forms mono-, di-, tri- or tetra-thiomolybdates [30], making $\mathrm{Cu}$ unavailable for absorption and utilization [33].

\section{Mineral levels in hair samples of lactating cows}

Hair samples collected during survey were analyzed for the same minerals as in feeds and forages. Mineral levels in hair must reflect the concentration and/or activity of the certain minerals in other parts of the body and reflect dietary mineral status of dairy cows [34]. The average levels of $\mathrm{Cu}$ and $\mathrm{Zn}$ in hair were 6.77 and $63.51 \mathrm{ppm}$, respectively (Table 6). When compared with critical levels for $\mathrm{Cu}(<10 \mathrm{ppm})$ and $\mathrm{Zn}$ $(<100 \mathrm{ppm}), 50$ and $100 \%$ cows showed sub-normal levels in hair samples indicating their dietary deficiency. It has been demonstrated in several studies that concentration of $\mathrm{Zn}$ in hair is correlated with dietary $\mathrm{Zn}$ intake $[35,36]$. Studies have shown the level of $\mathrm{Zn}$ in hair on normal diet to be $120-150 \mathrm{ppm}$ in dairy cows [1]. The Se level of the hair of cattle is a useful indicator of both the Se deficiency and Se toxicity [37]. Most studies had shown that dairy cows with hair values consistently below $0.25 \mathrm{ppm}$ probably need supplementation and that over $5 \mathrm{ppm}$ may lead to clinical signs of selenosis [38]. The average Se level in hair samples was $3.48 \mathrm{ppm}$, indicating the adequacy of the element in the ration of dairy cows.

Table 7 Minerals availability vis-à-vis requirement for dairy cow yielding $10 \mathrm{~kg}$ milk/day (4\% fat)

\begin{tabular}{|c|c|c|c|c|c|c|c|c|}
\hline Attributes & $\begin{array}{l}\mathrm{DMl} \\
(\mathrm{kg} / \mathrm{d})\end{array}$ & $\begin{array}{l}\text { Ca } \\
\text { (g) }\end{array}$ & $\begin{array}{l}P \\
(g)\end{array}$ & $\begin{array}{l}M g \\
(g)\end{array}$ & $\begin{array}{l}S \\
(g)\end{array}$ & $\begin{array}{l}\mathrm{Cu} \\
(\mathrm{mg})\end{array}$ & $\begin{array}{l}\mathrm{Zn} \\
(\mathrm{mg})\end{array}$ & $\begin{array}{l}\text { Co } \\
(\mathrm{mg})\end{array}$ \\
\hline Mineral requirement & 11.50 & 48.10 & 30.80 & 23 & 23 & 115 & 920 & 5.75 \\
\hline \multicolumn{9}{|l|}{ Mineral availability } \\
\hline Wheat bran (Triticum aestivum) & 2.5 & 7.0 & 2.25 & 2.75 & 2.0 & 6.70 & 25.53 & 0.45 \\
\hline Rice straw (Oryza sativa) & 4.0 & 12.8 & 3.20 & 4.64 & 4.4 & 11.96 & 107.12 & 1.44 \\
\hline Gram flour (Cicer arietinum) & 1.0 & 1.20 & 3.70 & 1.50 & 1.90 & 7.68 & 35.60 & 0.29 \\
\hline Local grasses & 2.0 & 12.80 & 7.20 & 5.60 & 3.60 & 21.74 & 87.01 & 0.72 \\
\hline Mustard cake (Brassica campestris) & 1.0 & 6.25 & 10.0 & 2.60 & 9.25 & 7.56 & 48.89 & 0.39 \\
\hline Gram chunni (Cicer arietinum) & 1.0 & 4.40 & 2.80 & 2.40 & 1.20 & 7.81 & 33.48 & 0.27 \\
\hline $\begin{array}{l}\text { Daily mineral availability from } \\
\text { traditional feeding }\end{array}$ & 11.50 & 44.45 & 29.15 & 19.5 & 22.35 & 63.45 & 337.63 & 3.56 \\
\hline Degree of deficiency (\%) & & 7.58 & 5.35 & 3.50 & 2.60 & 44.82 & 63.30 & 38.08 \\
\hline
\end{tabular}


Table 8 Mineral requirements for dairy cows

\begin{tabular}{lll}
\hline Particular & Calcium $(\mathrm{Ca})$ & Phosphorus $(\mathrm{P})$ \\
\hline Maintenance $(\mathrm{g})$ & 16 & 11 \\
Milk yield $(\mathrm{g} / \mathrm{kg})$ & 3.21 & 1.98 \\
$\begin{array}{l}\text { Mg and } \mathrm{S}: 0.20 \% \text { of DM } \\
\text { intake }\end{array}$ & Copper : $10 \mathrm{ppm}$ & $\begin{array}{l}\text { Manganese : } \\
\text { 40 ppm }\end{array}$ \\
$\mathrm{Na}: 0.18 \%$ of DM intake & Iron : $50 \mathrm{ppm}$ & Cobalt : $0.50 \mathrm{ppm}$ \\
$\mathrm{K}: 0.90 \%$ of DM intake & Zinc : $80 \mathrm{ppm}$ & Selenium : $0.30 \mathrm{ppm}$ \\
$\mathrm{Cl}: 0.25 \%$ of DM intake & lodine : & Chromium: $0.5 \mathrm{ppm}$ \\
& $0.60 \mathrm{ppm}$ & \\
\hline
\end{tabular}

\section{Daily mineral intake by a lactating cow}

The daily intake of different minerals by a HF crossbred cow (400 kg body weight) yielding $10 \mathrm{~kg}$ milk (4\% fat), with the prevailing feeding system in the surveyed area is presented in Table 7. Since mineral mixture supplementation was not being followed, so the intake of minerals through feeds, wet forages and dry roughages with the $60 \%$ bio-availability $[39,40]$ was taken as index of total mineral supply and compared with the recommended requirements to know the dietary mineral adequacy/deficiency. Ration of dairy cows was found to be deficient in $\mathrm{Ca}, \mathrm{P}, \mathrm{Mg}, \mathrm{S}, \mathrm{Cu}, \mathrm{Zn}$ and Co. Hence, it is necessary to supplement these minerals in the ration. It was observed that $\mathrm{K}, \mathrm{Na}, \mathrm{Mn}, \mathrm{Fe}$, Mo and Se in the ration of cows were found to be adequate. Supplementation of $\mathrm{Cu}$ and $\mathrm{Zn}$ in the form of chelates found to be more effective in curing problem of anestrous [41] and deficient trace minerals in the surveyed area may be supplemented in chelated form for better bio-availability and retention in the animal system.

\section{Formulation of area specific mineral mixture}

Information on the actual intake of each type of feeds and forage for a particular level of milk production was collected from each of the individual dairy farmer, to calculate intake of various mineral elements against the

Table 9 Area specific mineral mixture formulation for the state of Jharkhand

\begin{tabular}{llc}
\hline SI. No. & Characteristic & Requirement \\
\hline 1. & Calcium (\%), Min. & 21.0 \\
2. & Phosphorus (\%), Min. & 12.5 \\
3. & Magnesium (\%), Min. & 3.0 \\
4. & Sulphur (\%), Min. & 2.0 \\
5. & Copper (\%), Min. & 0.20 \\
6. & Zinc (\%), Min. & 1.40 \\
7. & Cobalt (\%), Min. & 0.016 \\
8. & lodine (\%), Min. & 0.026 \\
9. & Chromium (\%), Min. & 0.004 \\
\hline
\end{tabular}

Note: Values for requirement at SI. No. 1 to 9 are on dry matter basis requirement. Total mineral intake from feeds and forages was compared against the requirements on dry matter basis (Table 8), to identify quantitative deficiency and adequacy of minerals. Based on the degree of deficiency, specification of mineral mixture used in the state was modified with area specific mineral mixture, by incorporating deficient minerals at higher levels and reducing or excluding excess minerals from the formulation, for supplementing the dairy cows in the Jharkhand state (Table 9). To enhance the usefulness of mineral mixture, chromium was also incorporated in the formulation.

\section{Conclusions}

It was evident from the present study that majority of the dairy cows in Jharkhand state were deficient in $\mathrm{Ca}, \mathrm{P}$, $\mathrm{Mg}, \mathrm{S}, \mathrm{Cu}, \mathrm{Zn}$ and Co. Therefore, it is necessary to supplement these minerals in the ration of dairy cows by formulating area specific mineral mixture, having highly bio-available mineral salts. Deficient trace minerals, except Co, may be supplemented in the form of chelates, for better bio-availability and improving productivity, reproductive efficiency and productive life of dairy cows.

\section{Abbreviations \\ \%: Per cent; ADF: Acid detergent fibre; AIA: Acid insoluble ash; Ca: Calcium; CF: Crude fibre; Co: Cobalt; CP: Crude protein; Cu: Copper; EE: Ether extract; Fe: Iron; INAPH: Information Network for Animal Productivity and Health; K: Potassium; Kg: Kilo gram; Mg: Magnesium; Mn: Manganese; Mo: Molybdenum}

\section{Acknowledgements}

Financial assistance and necessary facilities provided by the management of National Dairy Development Board (NDDB), Anand, Gujarat, India for undertaking this study are gratefully acknowledged. Necessary support provided by officers of state animal husbandry department of Jharkhand is duly acknowledged

\section{Funding}

Financial assistance and necessary facilities in the designing the study, colleting and interpreting the research data have been provided by National Dairy Development Board, Anand, Gujarat, India.

\section{Availability of data and materials}

Readily reproducible materials described in the manuscript, including databases and all relevant raw data, freely. Freely available to any scientist wishing to use them, without breaching.

\section{Authors' contributions}

All the authors are participated in its design, coordination and write the manuscript. SS and AG assisted in the sample collection, processing, analysis, tabulation and interpretation of data. BMB and MRG have made necessary modifications in the manuscript. All the authors read and approved the final manuscript.

\section{Competing interests}

The authors declare that they have no competing interests.

\section{Consent for publication}

"Not applicable".

Ethical approval for the dairy cows for experimentation

The guidelines for animal experiments outlined by the Institutional Animal Ethics Committee which approved this study and the ethical guidelines/ protocols of the National Dairy Development Board, Anand, Gujarat, India, 
were followed during all the animal experimentations. There was no surgical procedure involved in the study. Names of the ethics committee that approved the study were Dr Shaileshkumar Kantilal Bhavsar, Dr B. Suresh, Dr Ramsharan S Gupta and Mrs Snehal Bhavsar.

\section{Ethics approval}

"Not applicable" as no animal and its tissue was used during study.

\section{Author details}

'Animal Nutrition Group, National Dairy Development Board (NDDB), Anand 388001, Gujarat, India. ${ }^{2}$ Head (Productivity Enhancement), Jharkhand Milk Federation, Ranchi, Jharkhand State, India.

\section{Received: 18 June 2016 Accepted: 3 December 2016}

Published online: 19 December 2016

\section{References}

1. Underwood EJ, Suttle NF. The Mineral Nutrition of Livestock. $3^{\text {rd }}$ edn. London: CAB International Publishing Co.; 1999.

2. Garg MR, Bhanderi BM, Sherasia PL. Assessment of macro and micro-minerals status of milch animals for developing area specific mineral mixture for Bharatpur district of Rajasthan. Anim Nutr Feed Tech. 2008;8:53-64.

3. McDowell LR. Minerals in animal and human nutrition. San Diego: Academic; 1992. p. 49-51.

4. Garg MR, Bhanderi BM, Sherasia PL. Trace minerals status of feeds and fodders in Junagadh district of Gujarat. Indian J Dairy Sci. 2002;55:154-58.

5. Bhanderi BM, Garg MR, Sherasia PL. Assessment of minerals status of dairy animals in South-West zone of Punjab. J Buffalo Sci. 2015;4:33-9.

6. Miles WH, McDowell LR. Mineral deficiencies in the llanos ranges. World Anim Rev. 1983;46:2-10.

7. Garg MR, Bhanderi BM, Sherasia PL. Macro and micro-mineral status of feeds and fodders in Kota district of Rajasthan. Indian J Anim Nutr. 2003;20:252-61.

8. Tourtelot HA. Geochemical surveys in United States in relation to health. Environmental Geochemistry and Health. London: Royal Society; 1980. pp. 113-126.

9. Garg MR, Sherasia PL, Phondba BT, Hossain HA. Effect of feeding a balanced ration on milk production, microbial nitrogen supply and methane emissions in field animals. Anim Prod Sci. 2014:54:1657-61.

10. Sherasia PL, Phondba BT, Hossain SA, Patel BP, Garg MR. Impact of feeding balanced rations on milk production, methane emission, metabolites and feed conversion efficiency in lactating cows. Indian J Anim Res. 2016;50(4):505-11.

11. NRC. Nutrient Requirements of Dairy Cattle. 7 revisedth ed. Washington: National Academy of Sciences; 2001.

12. AOAC. Official Methods of Analysis. 15th ed. Arlington: Association of Official Analytical Chemists; 1995.

13. Van Soest PJ, Robertson JB, Lewis BA. Methods of dietary fiber, neutra detergent fiber and non starch polysaccharides in relation to animal nutrition. J Dairy Sci. 1991;74:3583-97.

14. McDowell LR, Conrad JH, Glen Hembry F. Minerals for grazing ruminants in tropical regions. Animal Science Department, Centre for Tropical Agriculture, University of Florida. The US Agency for International Development and Caribbean Basin Advisory Group (CBAG).

15. Campbell MH, Miller JK, Schrick FN. Effect of additional cobalt, copper, manganese and zinc on reproduction and milk yield of lactating dairy cows receiving bovine somatotropin. J Dairy Sci. 1999;82:1019-22.

16. Snedecor GW, Cochran WG. Statistical Methods. 8th ed. New Delhi: lowa State University Press; 1994.

17. Anonymous. Progress Report Presented in the Meeting of Research Sub-Committee on Animal Production. Published by Animal Nutrition Research Department, Faculty of Veterinary Science \& A.H., Anand Agricultural University, Anand Campus, Anand-388001, Gujarat, 2006.

18. Bhanderi BM. Assessment of feeding practices and status of certain minerals in dairy animals of Jamnagar district of Gujarat State, MVSc Thesis. Anand: Anand Agricultural University; 2007.

19. Sherasia PL, Pandya PR, Parnerkar S, Patel DC, Bhanderi BM. Assessment of feeding practices and status of certain minerals of lactating cows in Porbandar district of Gujarat state. Indian J Dairy Sci. 2014;67(3):223-29.

20. Garg MR, Sherasia PL, Bhanderi BM, Phondba BT, Shelke SK, Makkar HPS. Effect of feeding nutritionally balanced rations on animal productivity, feed conversion efficiency, rumen microbial protein supply, parasitic load, immunity and enteric methane emissions of milch animals under field conditions. Anim Feed Sci Technol. 2013;179:24-35.

21. Garg MR, Bhanderi BM, Sherasia PL, Goswami A. Recent concepts in mineral nutrition. Proc. Global Animal Nutrition Conference-2014 on "Climate Resilient Livestock Feeding Systems for Global Food Security" organized jointly by the ANSI, NIANP, CLFMA of India and VIV India during April 20-22, 2014 at Bangalore, pp. 113-120.

22. Garg MR, Sherasia PL. Ration balancing at farmers' doorstep: a climate smart strategy for sustainable increase in livestock productivity in developing countries. CAB Rev. 2016;11(6):1-8.

23. Garg MR, Bhanderi BM, Shankhpal S, Goswami A, Sherasia PL. Impact of calf nutrition on overall production and productive life of an animal, including environment. CAB Rev. 2016;11(9):1-18.

24. Ramana J, Prasad CS, Gowda NKS, Ramachandra KS. Levels of micronutrients in soil, feed, fodder and animals of North East transition and dry zones of Karnataka. Indian J Anim Nutr. 2001;18:235-42.

25. Udar SA, Chopde S, Dhore RN. Mineral profile of soil, feeds and fodder and buffaloes in Western agro-climatic zone of Vidarbha. Anim Nutr Feed Tech. 2003;3:165-72.

26. Bhanderi BM, Goswami A, Garg MR. Mapping of macro and micro-mineral status of dairy cattle in Sabarkantha district of Gujarat. Livestock Res Intl. 2016:4(1):47-51.

27. Cuesta PA, McDowell LR, Kunkle WE, Bullock F, Drew A, Wilkinson NS, Martin FG. Seasonal variation of soil and forage mineral concentrations in North Florida. Comm Soil Sci Plant Anal. 1993;24:335-47.

28. Youssef FG, McDowell LR, Brathwaite RAl. The status of certain trace minerals and sulphur of some tropical grasses in Trinidad. Trop Agri. 1999; 76:57-62.

29. Yadav PS, Mandal AB, Dahiya DV. Feeding pattern and mineral status of buffaloes in Panipat district of Haryana state. Anim Nutr Feed Tech. 2002;2:127-38.

30. Suttle NF. The interactions between copper, molybdenum and sulphur in ruminant nutrition. Annual Rev Nutr. 1991;11:121-40.

31. NRC. National Research Council, Mineral Tolerances of Domestic Animals. Washington,: National Academy of Sciences; 1980.

32. Mandal AB, Yadav PS, Kapoor V. Mineral status of buffaloes under farm feeding condition of Faridabad district of Haryana state. Indian J Anim Nutr. 2004:21:104-10.

33. Nelson J. Review of trace mineral chelates and complexes available to the feed industry. Western Nutrition Conference, Winnipeg, Manitoba, September, 1988; P.1.

34. Combs DK. Hair analysis as an indicator of mineral status of livestock. J Anim Sci. 1987:65:1753-58.

35. Brown KH. Effect of infections on plasma zinc concentration and implications for zinc status assessment in low-income countries. Am J Clin Nutr. 1998;68(Suppl):425S-9S

36. Liu HY, Sun MH, Yang GQ, Jia CL, Zhang M, Zhu YJ, Zhang Y. Influence of different dietary zinc levels on cashmere growth, plasma testosterone level and zinc status in male Liaoning Cashmere goats. Anim Physiol Anim Nutr. 2015;99(5):880-86

37. Miller WJ. Dairy cattle feeding and nutrition. New York: Academic Press, Inc.; 1979

38. Williams SN, Frye TM, Scherf H, Frigg M. Vitamin E and selenium for ruminants. Proc. $4^{\text {th }}$ Annual Ruminant Nutrition Symposium. Gainesville, FL. 1993;90-108.

39. Miller ER. Techniques for determining bioavailability of trace elements. Proc. $6^{\text {th }}$ Annual International Minerals Conference, p. 23, St. Petersburg Beach, FL. 1983.

40. Spears JW. Trace mineral bioavailability in ruminants. J Nutr. 2003;133: 1506S.

41. Garg MR, Bhanderi BM, Gupta SK. Effect of supplementing certain chelated minerals and vitamins to overcome infertility in field animals. Indian J Dairy Sci. 2008b;61:181-84. 